International Mathematical Forum, Vol. 8, 2013, no. 37, 1793 - 1796

HIKARI Ltd, www.m-hikari.com

http://dx.doi.org/10.12988/imf.2013.310195

\title{
On the Arithmetic Properties of Polyadic Integers
}

\author{
V. G. Chirskii \\ Moscow State Lomonosov University, Russia
}

Copyright (C) 2013 V. G. Chirskii. This is an open access article distributed under the Creative Commons Attribution License, which permits unrestricted use, distribution, and reproduction in any medium, provided the original work is properly cited.

\begin{abstract}
The paper presents a classification of polyadic integers with respect to their arithmetic properties.
\end{abstract}

Keywords: polyadic integers, algebraic numbers, transcendental numbers.

We begin with a brief introduction to the theory of polyadic integers. Let $K$ be a commutative ring. A mapping $v$ of $K$ into non-negative real numbers is called a non-archimedean pseudo-valuation of $K$ if it has the following properties:

1. $v(a) \geq 0$ for all $a \in K, v(a)=0$ iff $a=0 \in K$.

2. $v(a b) \leq v(a) v(b)$ for all $a, b \in K$.

3. $v(a \pm b) \leq \max (v(a), v(b))$ for all $a, b \in K$.

If for all $a, b \in K$ the stronger condition $v(a b)=v(a) v(b)$ holds, then $v$ is called a valuation. For a prime $p$ the $p$-adic valuation $|\alpha|_{p}$ of an element $\alpha \in \mathbb{Q}$ is defined as follows. If $a \in \mathbb{Z}$ is divisible by $p^{f}$ and not divisible by $p^{f+1}$, then $|a|_{p}=p^{-f}$. For $\alpha=a /_{b}, a \in \mathbb{Z}, b \in \mathbb{N}$ we have $|\alpha|_{p}=|a|_{p} /|b|_{p}$. As usual, $\mathbb{Q}_{p}$ denotes the corresponding completion of $\mathbb{Q}$. It is the field of $p$-adic numbers, and $\mathbb{Z}_{p}$ denotes the ring of $p$-adic integers which satisfy the inequality $|\alpha|_{p} \leq 1$. For $g=p_{1}^{r_{1}} \ldots p_{s}^{r_{s}}$, where $p_{i}$ are primes and $r_{i}$ are positive integers we can consider the $g$ - adic pseudo-valuation which is defined in a similar way (cf. [7]). 
The corresponding completion is denoted by $\mathbb{Q}_{g}$. A well-known theorem by K. Hensel (cf. [7]) asserts that $\mathbb{Q}_{g}$ is a direct sum of the fields $\mathbb{Q}_{p_{1}}, \ldots, \mathbb{Q}_{p_{s}}$, so any element $A \in \mathbb{Q}_{g}$ can be expressed as $A=\left(A_{1}, \ldots, A_{s}\right)$ with $A_{i} \in \mathbb{Q}_{p_{i}}$ and for any polynomial $P(x) \in \mathbb{Z}[x]$ one has $P(A)=\left(P\left(A_{1}\right), \ldots, P\left(A_{s}\right)\right)$. Recall that $\alpha \in K$ is called algebraic (over $\mathbb{Q}$ ) if there exists a nonzero polynomial $P(x) \in \mathbb{Z}[x]$ such that $P(\alpha)=0$. Otherwise it is called transcendental. Therefore $A \in \mathbb{Q}_{g}$ is algebraic iff $A_{i} \in \mathbb{Q}_{p_{i}}, i=1, \ldots, s$ are algebraic.

We introduce a topology in the ring $\mathbb{Z}$ by considering the set of all ideals $(m)$ as the system of vicinities of zero. Addition and multiplication are continuous with respect to this topology. The completion of this topological ring is called the ring of polyadic integers. ( The detailed descriptions of the construction of polyadic numbers are presented in [8]). The elements of this ring have canonical representations of the form $\sum_{n=0}^{\infty} a_{n} \cdot n !, a_{n} \in\{0,1, \ldots, n\}$. A series of this form converges in any $\mathbb{Q}_{p}$ and, for example, in any $\mathbb{Q}_{p}$ we have $\sum_{n=1}^{\infty} n \cdot n !=-1$.

One can prove that the ring of polyadic integers is a prime product of the rings $\mathbb{Z}_{p}$ over all primes $p$. Therefore any polyadic integer can be expressed as $A=\left(A_{1}, \ldots, A_{s}, \ldots\right)$ where the components $A_{s}$, belong to $\mathbb{Z}_{p_{s}}$, e.g. $\sum_{n=1}^{\infty} n \cdot n !=$ $(-1, \ldots,-1, \ldots)$. This remark allows us to give the following definition: a polyadic integer $A$ is algebraic, if there exists a polynomial $P(x) \in \mathbb{Z}[x]$ such that $P(A)=0($ where $0=(0, \ldots, 0, \ldots))$, in other words, if for any $s$ one has $P\left(A_{s}\right)=0$ in $\mathbb{Z}_{p_{s}}$. In terms of [1], [3] it means that $A$ satisfies a global relation.

We call the polyadic integer $A$ transcendental, if for any nonzero polynomial $P(x) \in \mathbb{Z}[x]$ there exists at least one prime $p$ such that $P\left(A_{p}\right) \neq 0$ in $\mathbb{Q}_{p}$. We call the polyadic integer $A$ infinitely transcendental, if for any nonzero polynomial $P(x) \in \mathbb{Z}[x]$ there exist infinitely many primes $p$ such that $P\left(A_{p}\right) \neq 0$ in $\mathbb{Q}_{p}$. At last, a polyadic integer is globally transcendental, if for any nonzero polynomial $P(x) \in \mathbb{Z}[x]$ and for all primes $p$ the inequality $P\left(A_{p}\right) \neq 0$ holds in $\mathbb{Q}_{p}$. Of course, globally transcendental polyadic integers form a subset of infinitely transcendental polyadic integers which, in turn, form a subset of transcendental polyadic integers. Hensel's theorem mentioned above implies that there exist transcendental polyadic integers, which are not infinitely transcendental, and there exist infinitely transcendental polyadic integers, which are not globally transcendental.

Let now $\sum_{n=1}^{\infty} n !=A$. From [1], [4] it immediately follows that $A$ is at least infinitely transcendental. A very interesting problem is to prove that this polyadic number is globally transcendental. Using theorem from [1] and results 
from [2], [9] one can easily prove that a lot of numbers, which are the values of generalized hypergeometric series are infinitely transcendental and even are infinitely algebraically independent( the definition of this notion is in analogy to the definition of infinitely transcendental polyadic integers, one must only consider $\left.P\left(x_{1}, \ldots, x_{n}\right) \in \mathbb{Z}\left[x_{1}, \ldots, x_{n}\right]\right)$. It is worth to note ,cf [6], that for any polynomial $P(x) \in \mathbb{Z}[x]$ we have $\sum_{n=0}^{\infty} P(n) n !=C+D \sum_{n=1}^{\infty} n$ ! with certain $C, D \in \mathbb{Z}$.

In [5] it is proved that there exist globally transcendental and globally algebraically independent polyadic integers.

\section{References}

[1] D.Bertrand, V.Chirskii, D.Yebbou, Effective estimates for global relations on Euler-type series, Ann. Fac. Sci. Toulouse, v.XIII, 2(2004),241-260.

[2] F.Beukers,W. D. Brownawell, G.Heckmann, Siegel normality, Annals of Mathematics, v.127(1988),279-308.

[3] E.Bombieri, On G-functions, Recent progress in analytic number theory, Academic Press,v.2, London(1981), 1-68.

[4] V.G. Chirskii, On global relations, Matem. Zametki (rus.),v.48, 2(1990), 123 127.

[5] V.G. Chirskii, On the series which are algebraically independent in all local fields, Vestnik Moskov. Univer, Ser 1, (rus.) 3(1994), 93-95.

[6] V.G. Chirskii. Arithmetic properties of certain polyadic series, Vestnik Moskov. Univer, Ser 1, (rus.) 5(2012), 52-54.

[7] K.Mahler, p-adic numbers and their functions, Cambridge Univ. Press, London, New York (1980).

[8]H. Prüfer, Neue Begründung der algebraischen Zahlentheorie, Math. Ann. V.94, 3-4(1925), 198-243. 
[9] V.Kh. Salikhov, Algebraic independence of values of hypergeometric Efunctions, Soviet Math. Dokl. 40(1990), 71-74.

Received: October 21, 2013 